\title{
Acesso de pacientes com necessidades especiais em CEOs de Sergipe
}

\author{
Access for patients with special needs in Sergipe's CEOs \\ Acceso para pacientes con necesidades especiales en los CEOs de Sergipe
}

Recebido: 12/03/2021 | Revisado: 18/03/2021 | Aceito: 19/03/2021 | Publicado: 27/03/2021

\author{
Rosana Apolonio Reis Andrade \\ ORCID: https://orcid.org/0000-0003-3195-0212 \\ Universidade Federal de Sergipe, Brasil \\ E-mail: rosana.apolonio@gmail.com \\ Amanda Caroline Nascimento Meireles \\ ORCID: https://orcid.org/0000-0001-6007-9257 \\ Universidade Federal de Sergipe, Brasil \\ E-mail: c4roline21@gmail.com \\ Alisson Augusto Gois de Almeida \\ ORCID: https://orcid.org/0000-0001-6027-946X \\ Universidade Federal de Sergipe, Brasil \\ E-mail: alisonalmeida.odo@gmail.com \\ Regiane Cristina do Amaral \\ ORCID: https://orcid.org/0000-0002-9191-0960 \\ Universidade Federal de Sergipe, Brasil \\ E-mail: amaralre@yahoo.com.br
}

\begin{abstract}
Resumo
A Política Nacional de Saúde Bucal foi instituída como ferramenta para possibilitar a integralidade das ações em saúde da população. Os Centros de Especialidades Odontológicas (CEO) se constituem como estabelecimentos essenciais, dentro da atenção secundária, para a continuidade da prestação de oferta dos serviços odontológicos especializados e efetivação do cuidado em saúde bucal. Assim, o presente estudo teve por objetivo avaliar a correlação entre as características socioeconômicas e demográficas dos pacientes com necessidades especiais (PNE) em Sergipe e acesso aos serviços dos CEOs Estaduais de Sergipe. Foram utilizados dados da FUNESA (Fundação Estadual de Saúde), e-Gestor e CECAD (Consulta, Seleção e Extração de Informações do CadÚnico). Foram avaliados 7 CEOs Estaduais (2018) e verificou-se correlação estatisticamente significativa para: cobertura de saúde bucal e pacientes especiais que recebem bolsa família ( $\mathrm{p}=0,0007 ; \mathrm{r}=-0.397$ ); cobertura de saúde bucal e distância do município até a sede do CEO ( $\mathrm{p}=0.0322 ; \mathrm{r}=-0.2563)$; cobertura de saúde bucal e população do município que tem renda mensal per capta até $\mathrm{R} \$ 178,00(\mathrm{p}=<0.0001 ; \mathrm{r}=-0.5418)$. A maior parte do atendimento dos pacientes ocorre dentro do próprio município sede. Há dificuldade de acesso do paciente com necessidades especiais em termos de referenciamento e assistência em percentual de cobertura de saúde bucal. O baixo percentual de cobertura de saúde bucal e de referenciamento dificultam o acesso dos PNE à assistência odontológica. As condições socioeconômicas e demográficas interferem no acesso dos PNE aos CEOs em Sergipe.
\end{abstract}

Palavras-chave: Odontologia; Odontologia em saúde pública; Saúde pública; Pessoas com deficiência.

\begin{abstract}
The National Oral Health Policy was instituted as a tool to enable comprehensive health actions for the population. The Dental Specialty Centers (CEO) are essential establishments, within secondary care, for the continuity of the provision of specialized dental services and the effectiveness of oral health care. Thus, the present study aimed to assess the correlation between the socioeconomic and demographic characteristics of patients with special needs (PNE) in Sergipe and access to the services of State CEOs of Sergipe. Data from FUNESA (State Health Foundation), e-Manager and CECAD (Consultation, Selection and Extraction of Information from CadÚnico) were used. Seven State CEOs (2018) were evaluated and there was a statistically significant correlation for: oral health coverage and special patients receiving family allowance $(\mathrm{p}=0.0007 ; \mathrm{r}=-0.397)$; oral health coverage and distance from the municipality to the CEO's headquarters $(\mathrm{p}=0.0322 ; \mathrm{r}=-0.2563)$; oral health coverage and population of the municipality with monthly income per capita up to R $\$ 178.00(p=\langle 0.0001 ; r=-0.5418)$. Most of the care provided to patients takes place within the host city itself. There is difficulty in accessing patients with special needs in terms of referral and assistance in percentage of oral health coverage. The low percentage of oral health coverage and referral makes it difficult for PNE to access dental care. Socioeconomic and demographic conditions interfere with PNE access to CEOs in Sergipe.
\end{abstract}

Keywords: Dentistry; Public health dentistry; Public health; Disabled persons. 


\begin{abstract}
Resumen
La Política Nacional de Salud Bucal se instituyó como una herramienta para posibilitar acciones integrales de salud para la población. Los Centros de Especialidades Dentales (CEO) son establecimientos imprescindibles, dentro de la atención secundaria, para la continuidad de la prestación de servicios dentales especializados y la eficacia de la atención de la salud bucal. Así, el presente estudio tuvo como objetivo evaluar la correlación entre las características socioeconómicas y demográficas de los pacientes con necesidades especiales (PNE) en Sergipe y el acceso a los servicios de los directores generales estatales de Sergipe. Se utilizaron datos de FUNESA (Fundación Estatal de Salud), e-Manager y CECAD (Consulta, Selección y Extracción de Información de CadÚnico). Se evaluaron siete directores generales estatales (2018) y hubo una correlación estadísticamente significativa para: cobertura de salud bucal y pacientes especiales que reciben asignación familiar ( $\mathrm{p}=0,0007 ; \mathrm{r}=-0,397)$; cobertura de salud bucal y distancia del municipio a la sede del CEO ( $\mathrm{p}=0.0322 ; \mathrm{r}=-0.2563)$; cobertura de salud bucal y población del municipio con ingreso mensual per cápita hasta $\mathrm{R} \$ 178,00(\mathrm{p}=<0,0001 ; \mathrm{r}=-0,5418)$. La mayor parte de la atención que se brinda a los pacientes se lleva a cabo dentro de la propia ciudad de acogida. Existe dificultad para acceder a pacientes con necesidades especiales en términos de derivación y asistencia en porcentaje de cobertura de salud bucal. El bajo porcentaje de cobertura y derivación de salud bucal dificulta el acceso de la PNE a la atención odontológica. Las condiciones socioeconómicas y demográficas interfieren con el acceso del PNE a los directores ejecutivos en Sergipe.
\end{abstract}

Palabras clave: Odontología; Odontología en salud pública; Salud pública; Personas con discapacidad.

\title{
1. Introdução
}

A atenção à saúde bucal no âmbito do Sistema Único de Saúde (SUS) foi reorganizada a partir da Política Nacional de Saúde Bucal (PNSB) que propôs uma concepção de saúde centrada na promoção de saúde e intervenção sobre os fatores de risco (Brasil, 2004; Costa et al., 2012). Assim, atendendo ao princípio da integralidade, às diretrizes da PNSB estabeleceram a ampliação e qualificação da oferta de serviços odontológicos especializados por meio da implantação dos Centros de Especialidades Odontológicas (CEOs) através da Portaria nº 599, de 23 de março de 2006 (Brasil, 2008; Brasil, 2006). Afinal, havia a necessidade do estabelecimento de um sistema de referência e contrarreferência entre a atenção básica e assistência especializada em saúde bucal (Brasil, 2004).

No Brasil, a atenção básica em saúde bucal possui diferentes percentuais de cobertura entre os municípios podendo isso atuar como dificultador no processo de referenciamento para os serviços especializados. Os Centros de Especialidades Odontológicas (CEOs) são considerados serviços necessários para complementariedade do atendimento para além da atenção primária. O tratamento realizado nos CEOs deve ser uma continuidade do que é ofertado nas unidades básicas de saúde (UBS) pelas Equipes de Saúde Bucal (ESB). Deve haver interface com a rede de atenção básica dos municípios, a qual deve funcionar como porta de entrada para essa rede de cuidados (Brasil, 2017).

Os CEOs são responsáveis por oferecer serviços odontológicos especializados à população. Entre as especialidades obrigatórias estão: diagnóstico bucal (ênfase em diagnóstico e detecção de câncer bucal), periodontia especializada, cirurgia oral menor dos tecidos moles e duros, endodontia e atendimento a pacientes especiais (Brasil, 2006).

$\mathrm{Na}$ odontologia, é considerado paciente com necessidades especiais todo usuário que apresente uma ou mais limitações, temporária(s) ou permanente(s), de ordem mental, física, sensorial, emocional, de crescimento ou médica, que o impeça(m) de ser submetido a uma situação odontológica convencional (Brasil, 2008).

No Estado de Sergipe, em maio de 2020, os CEOs de acordo com o cadastrado no CNES (Cadastro de Estabelecimento de Saúde) estavam habilitados em 02 tipos de modalidades: 09 CEOs tipo II e do 03 do tipo III. Sendo no total 12 CEOs, 5 deles de administração municipal (Canindé de São Francisco, Estância, Lagarto, Aracaju, Nossa Senhora do Socorro) e 7 de administração estadual (Boquim, Capela, Laranjeiras, Nossa Senhora da Glória, Propriá, Tobias Barreto e São Cristóvão). De acordo com a capacidade instalada possuem 03 classificações: CEO Tipo I (mínimo de 03 cirurgiões-dentistas), CEO Tipo II (04 ou mais dentistas) e CEO Tipo III (07 ou mais dentistas) (Brasil, 2020A). 
De acordo com protocolo da FUNESA, os CEOs estaduais foram implantados em municípios-sede para contemplar à disposição de oferta dos demais municípios nas 7 regiões de saúde em Sergipe. Considerando os princípios da regionalização, localização geográfica e organização estrutural (Sergipe, 2019). Em relação a cobertura da atenção básica, em setembro de 2019, de acordo com dados do Ministério da Saúde (MS), Sergipe possuía 684 ESF e 504 ESB, correspondendo respectivamente a 87,3\% e 70,2\% de cobertura populacional (Brasil, 2020 B).

No Estado de Sergipe, os CEOs Estaduais prestam serviços a 70 municípios sergipanos sendo assim responsáveis pela maior cobertura de referenciamento para a atenção secundária (93,33\%, equivalente a 1.263 .029 pessoas). Este conjunto da rede básica de saúde, rede de atenção especializada, acrescidos da rede hospitalar devem se organizar de forma complementar a partir dos equipamentos assistenciais e respectivas ofertas de serviços numa conformação de uma rede assistencial de saúde como forma de garantir uma atenção contínua e integral a população (Brasil, 2014). O atendimento ao paciente com necessidades especiais ainda se dá no Brasil por meio das Redes de Atenção à Saúde (RAS) que são arranjos organizativos de ações e serviços de saúde, de diferentes densidades tecnológicas que, integradas por meio de sistemas de apoio técnico, logístico e de gestão, buscam garantir a integralidade do cuidado (Mendes, 2011).

De acordo com os dados da última Pesquisa Nacional de Atenção Domiciliar (PNAD) realizada em 2013, estimou-se que $80 \%$ da população brasileira é SUS-dependente. Segue-se a mesma tendência em relação aos estados do Nordeste, haja vista a baixa renda per capita da população. Em Sergipe, tem-se também uma equivalência percentual da população que necessita do SUS para atendimento as suas necessidades de saúde (IBGE, 2013).

Como forma de sistematizar os dados a respeito desse grupo populacional foi criado no Brasil o CadÚnico (Cadastro Único de Programas Sociais), através do Decreto Presidencial nº 6.135, de 26 de junho de 2007, com o objetivo de identificar todas as famílias de baixa renda para inclusão em programas de assistência social e redistribuição de renda. Em Sergipe existem 48.5370 famílias cadastradas, das quais $60 \%$ possuem renda per capta de até R $\$ 89,00$ mensais (Brasil, 2020 C; Brasil, 2007).

De acordo com o estudo de Carreiro e colaboradores (2019), a população de baixa renda é considerada dentro dos grupos com maiores vulnerabilidades social e com maior dificuldade de acesso aos serviços e tratamentos odontológicos. Por conseguinte, a atenção básica, através dos profissionais das equipes de saúde da família (ESF) e equipes de saúde bucal (ESB), são considerados com fundamentais para o referenciamento dos pacientes e continuidade do tratamento na atenção especializada.

Assim, o objetivo deste estudo foi avaliar a correlação entre as características socioeconômicas e demográficas dos pacientes com necessidades especiais em Sergipe e acesso aos serviços dos CEOs Estaduais de Sergipe.

\section{Metodologia}

O presente estudo foi submetido ao comitê de ética em Pesquisa da Universidade Federal de Sergipe, de acordo com a Resolução 466/2012, do Conselho Nacional de Saúde (CAEE: 91726818.8.0000.5546).

Esse estudo foi realizado por meio da coleta de dados secundários (estudo ecológico) registrados pelo Sistema CECAD 2.0 - Sistema de Consulta, Seleção e Extração de Informações do CadÚnico, afim de verificar condições sócio econômicas dos municípios do Estado do Sergipe. Esse sistema constitui uma ferramenta online para consulta pública que apresenta dados de caracterização socioeconômica das famílias incluídas no CadÚnico e famílias beneficiárias do Bolsa Família. 
O CECAD 2.0 em relação as pessoas com necessidades especiais apresentam uma categorização específica: cegueira, Síndrome de Down, deficiência física, deficiência mental/intelectual, surdez leve/moderada, surdez severa/profunda, transtorno/doença mental e baixa visão.

Foi realizada também a coleta de dados secundários do sistema e-Gestor Atenção Básica (e-Gestor), uma plataforma web que reúne dados e relatórios sobre a cobertura e funcionamento da atenção básica em Saúde no território brasileiro.

Os CEOs Estaduais se situam nos municípios de Boquim, Capela, Laranjeiras, Nossa Senhora da Glória (Glória), Propriá, São Cristóvão e Tobias Barreto. Os CEOs estão classificados como do tipo II, com exceção do localizado em São Cristóvão que é do tipo III.

Para isso utilizou-se a ferramenta Microsoft Excel. Foi realizado teste de normalidade das variáveis e correlação de Pearson, utilizando o programa estatístico SPSS 21.0.

\section{Resultados}

Foi realizado correlação (Correlação de Pearson) entre as variáveis como percentual de cobertura de saúde bucal (atenção básica), pacientes especiais que recebem bolsa família, distância do município até a sede do CEO e população do município que tem renda mensal per capta até $\mathrm{R} \$ 178,00$, total da população e taxa de pobreza.

Verificou-se correlação estatisticamente significativa para: cobertura de saúde bucal e pacientes especiais que recebem bolsa família ( $\mathrm{p}=0,0007 ; \mathrm{r}=-0.397$ ); cobertura de saúde bucal e distância do município até a sede do CEO ( $\mathrm{p}=0.0322$; $\mathrm{r}=-0.2563$ ); cobertura de saúde bucal e população do município que tem renda mensal per capta até $\mathrm{R} \$ 178,00$ ( $\mathrm{p}=<0.0001$; $\mathrm{r}=-0.5418)$; cobertura de saúde bucal e total da população $(\mathrm{p}=<0.0001 ; \mathrm{r}=-0.489)$; pacientes especiais que recebem bolsa família e população do município que tem renda mensal per capta até $\mathrm{R} \$ 178,00(\mathrm{p}=<0.0001 ; \mathrm{r}=0.8633)$; pacientes especiais que recebem bolsa família e total da população ( $\mathrm{p}=<0.0001 ; \mathrm{r}=0.8249$ ); população do município que tem renda mensal per capta até $\mathrm{R} \$ 178,00$ e total da população $(\mathrm{p}=<0.0001 ; \mathrm{r}=0.9165)$; total da população e taxa de pobreza $(\mathrm{p}=0.0068 ; \mathrm{r}=$ $0.3207)$.

Assim, quanto menor o percentual de cobertura de saúde, maior o número de pessoas com bolsa família, ou seja, maior número de pessoas com baixa renda. A exemplo disso tem-se o município de Poço Redondo com percentual de saúde bucal de 58,8\% e 241 pessoas com necessidades especiais que tem bolsa família, 20.554 pessoas com renda per capita de até $\mathrm{R} \$ 178,00$ e com distância de $70 \mathrm{~km}$ até o CEO.

Foi verificado que há variações na cobertura de saúde bucal dos municípios estudados. Há municípios com 100\% de percentual de cobertura de saúde bucal que não fazem referenciamento da Atenção Básica (primária) para os CEOs (secundárias) (Figuras 1). 
Tabela 1: Cobertura de saúde bucal de atenção básica (\%), número de pacientes especiais que recebem bolsa família, distância do município até o CEO e população com renda mensal per capita até R\$178,00, em 2019, total de população e taxa de pobreza.

\begin{tabular}{|c|c|c|c|c|c|c|}
\hline Municípios & Cobert SB \% & $\begin{array}{l}\text { Pacientes } \\
\text { especiais que } \\
\text { recebem bolsa } \\
\text { família (n) }\end{array}$ & $\begin{array}{l}\text { Distância } \\
\text { Sede }(\mathbf{k m})\end{array}$ & $\begin{array}{c}\text { População } \\
\text { com renda } \\
\text { mensal per } \\
\text { capita até } \mathbf{R} \$ \\
178,00\end{array}$ & $\begin{array}{c}\text { Total } \\
\text { população } \\
\text { (IBGE/2019) }\end{array}$ & Taxa pobreza \\
\hline *Boquim & 75,75 & 221 & 0 & 12025 & 26816 & 44,84 \\
\hline Arauá & 100 & 76 & 18 & 5880 & 10056 & 58,47 \\
\hline Salgado & 84,13 & 171 & 29 & 9816 & 19998 & 49,08 \\
\hline Pedrinhas & 100 & 88 & 8,2 & 4801 & 9602 & 50,00 \\
\hline Cristinápolis & 100 & 213 & 52 & 11039 & 17874 & 61,76 \\
\hline Indiaroba & 97,12 & 109 & 59 & 9995 & 17957 & 55,66 \\
\hline $\begin{array}{r}\text { Santa Luzia do } \\
\text { Itanhy }\end{array}$ & 100 & 109 & 38 & 8849 & 14035 & 63,05 \\
\hline Umbaúba & 55,13 & 169 & 36 & 12286 & 25294 & 48,57 \\
\hline *Laranjeiras & 100 & 274 & 0 & 11012 & 29826 & 36,92 \\
\hline Divina Pastora & 100 & 26 & 22 & 2403 & 5138 & 46,77 \\
\hline Riachuelo & 100 & 63 & 13 & 4986 & 10213 & 48,82 \\
\hline $\begin{array}{r}\text { Santa Rosa de } \\
\text { Lima }\end{array}$ & 100 & 17 & 25 & 2226 & 3913 & 56,89 \\
\hline Maruim & 77,83 & 58 & 13 & 7052 & 17213 & 40,97 \\
\hline $\begin{array}{r}\text { Rosário do } \\
\text { Catete }\end{array}$ & 100 & 68 & 21 & 4986 & 10855 & 45,93 \\
\hline $\begin{array}{r}\text { Santo Amaro } \\
\text { das Brotas }\end{array}$ & 100 & 67 & 22 & 2717 & 12102 & 22,45 \\
\hline Pirambu & 100 & 28 & 51 & 4857 & 9280 & 52,34 \\
\hline *Capela & 100 & 184 & 0 & 16930 & 34213 & 49,48 \\
\hline Carmópolis & 100 & 73 & 25 & 8179 & 16634 & 49,17 \\
\hline Cumbe & 100 & 13 & 35 & 2072 & 3987 & 51,97 \\
\hline $\begin{array}{l}\text { General } \\
\text { Maynard }\end{array}$ & 100 & 149 & 31 & 1365 & 3346 & 40,79 \\
\hline $\begin{array}{r}\text { Nossa Sra das } \\
\text { Dores }\end{array}$ & 100 & 231 & 19 & 13727 & 26629 & 51,55 \\
\hline Siriri & 100 & 43 & 13 & 4205 & 8893 & 47,28 \\
\hline Japaratuba & 95,28 & 61 & 20 & 8340 & 18743 & 44,50 \\
\hline
\end{tabular}


Research, Society and Development, v. 10, n. 3, e56610313829, 2021

(CC BY 4.0) | ISSN 2525-3409 | DOI: http://dx.doi.org/10.33448/rsd-v10i3.13829

\begin{tabular}{|c|c|c|c|c|c|c|}
\hline *Propriá & 93,37 & 250 & 0 & 11147 & 29626 & 37,63 \\
\hline $\begin{array}{r}\text { Amparo de São } \\
\text { Francisco }\end{array}$ & 100 & 14 & 20 & 1052 & 2374 & 44,31 \\
\hline Aquidabã & 48,27 & 96 & 42 & 8694 & 21563 & 40,32 \\
\hline Brejo Grande & 100 & 69 & 66 & 4971 & 8309 & 59,83 \\
\hline Canhoba & 100 & 21 & 22 & 3051 & 4008 & 76,12 \\
\hline $\begin{array}{r}\text { Cedro de São } \\
\text { João }\end{array}$ & 100 & 48 & 15 & 2402 & 5897 & 40,73 \\
\hline Japoatã & 100 & 70 & 18 & 9650 & 13434 & 71,83 \\
\hline Ilha das Flores & 100 & 70 & 51 & 5277 & 8520 & 61,94 \\
\hline $\begin{array}{r}\text { Malhada dos } \\
\text { Bois }\end{array}$ & 100 & 35 & 25 & 1881 & 3682 & 51,09 \\
\hline Neópolis & 73,66 & 98 & 43 & 9244 & 18716 & 49,39 \\
\hline $\begin{array}{r}\text { Nossa Senhora } \\
\text { de Lourdes }\end{array}$ & 100 & 47 & 35 & 3363 & 6486 & 51,85 \\
\hline Pacatuba & 100 & 107 & 40 & 7553 & 14428 & 52,35 \\
\hline $\begin{array}{r}\text { Santana do São } \\
\text { Francisco }\end{array}$ & 100 & 46 & 39 & 5603 & 7780 & 72,02 \\
\hline São Francisco & 100 & 18 & 21 & 1693 & 37224 & 4,55 \\
\hline Muribeca & 100 & 45 & 31 & 3875 & 7625 & 50,82 \\
\hline Telha & 100 & 25 & 9,4 & 2004 & 3227 & 62,10 \\
\hline *São Cristóvão & 62 & 508 & 0 & 34639 & 90072 & 38,46 \\
\hline Areia Branca & 72,57 & 101 & 47 & 7792 & 18542 & 42,02 \\
\hline $\begin{array}{r}\text { Barra dos } \\
\text { Coqueiros }\end{array}$ & 100 & 230 & 30 & 9107 & 30407 & 29,95 \\
\hline Campo do Brito & 100 & 111 & 75 & 7546 & 18109 & 41,67 \\
\hline Carira & 15,88 & 132 & 123 & 10898 & 22082 & 49,35 \\
\hline Frei Paulo & 100 & 144 & 86 & 7605 & 15421 & 49,32 \\
\hline Itabaiana & 50,05 & 317 & 67 & 33701 & 95427 & 35,32 \\
\hline $\begin{array}{r}\text { Itaporanga } \\
\text { D'ajuda }\end{array}$ & 100 & 317 & 21 & 16747 & 34356 & 48,75 \\
\hline Macambira & 100 & 83 & 84 & 3819 & 6919 & 55,20 \\
\hline Malhador & 78,69 & 153 & 62 & 6678 & 12618 & 52,92 \\
\hline Moita Bonita & 91,41 & 39 & 76 & 9608 & 11335 & 84,76 \\
\hline Pedra Mole & 100 & 17 & 109 & 1891 & 3261 & 57,99 \\
\hline
\end{tabular}


Research, Society and Development, v. 10, n. 3, e56610313829, 2021

(CC BY 4.0) | ISSN 2525-3409 | DOI: http://dx.doi.org/10.33448/rsd-v10i3.13829

\begin{tabular}{|c|c|c|c|c|c|c|}
\hline Pinhão & 100 & 26 & 110 & 3197 & 6576 & 48,62 \\
\hline Ribeirópolis & 90,67 & 31 & 88 & 6920 & 18652 & 37,10 \\
\hline São Domingos & 31,18 & 69 & 68 & 4877 & 11137 & 43,79 \\
\hline $\begin{array}{l}* \text { Tobias } \\
\text { Barreto }\end{array}$ & 51,5 & 223 & 0 & 23904 & 52191 & 45,80 \\
\hline Poço Verde & 71,23 & 241 & 55 & 11468 & 23728 & 48,33 \\
\hline $\begin{array}{r}\text { Riachão do } \\
\text { Dantas }\end{array}$ & 52,27 & 146 & 34 & 11284 & 19805 & 56,98 \\
\hline Simão Dias & 66,69 & 325 & 78 & 28407 & 40487 & 70,16 \\
\hline Itabaianinha & 68,73 & 219 & 31 & 22972 & 41928 & 54,79 \\
\hline Tomar do Geru & 100 & 150 & 45 & 7108 & 13536 & 52,51 \\
\hline $\begin{array}{r}\text { *Nossa } \\
\text { Senhora da } \\
\text { Glória }\end{array}$ & 75,59 & 195 & 0 & 18120 & 36924 & 49,07 \\
\hline Feira Nova & 100 & 43 & 14 & 3750 & 5584 & 67,16 \\
\hline Gracho Cardoso & 100 & 45 & 28 & 4617 & 5818 & 79,36 \\
\hline Gararu & 98,23 & 74 & 70 & 7231 & 11604 & 62,31 \\
\hline Itabi & 70,11 & 38 & 47 & 2366 & 4903 & 48,26 \\
\hline $\begin{array}{r}\text { Monte Alegre de } \\
\text { Sergipe }\end{array}$ & 68,45 & 134 & 29 & 10362 & 15031 & 68,94 \\
\hline Poço Redondo & 58,85 & 241 & 57 & 20554 & 34775 & 59,11 \\
\hline Porto da Folha & 52,11 & 141 & 70 & 17210 & 28596 & 60,18 \\
\hline $\begin{array}{r}\text { São Miguel do } \\
\text { Aleixo }\end{array}$ & 100 & 20 & 23 & 2141 & 3930 & 54,48 \\
\hline $\begin{array}{r}\text { Nossa Senhora } \\
\text { Aparecida }\end{array}$ & 100 & 52 & 22 & 4059 & 8796 & 46,15 \\
\hline
\end{tabular}

*Municípios-sede dos CEOs estaduais. Fonte: CECAD-IBGE/E-SUS (2019). 
Research, Society and Development, v. 10, n. 3, e56610313829, 2021

(CC BY 4.0) | ISSN 2525-3409 | DOI: http://dx.doi.org/10.33448/rsd-v10i3.13829

Figura 1: Percentual de encaminhamentos dos pacientes para atendimentos nos Centros de Especialidades Estaduais de Sergipe, 2018. *Acima de 4\%.

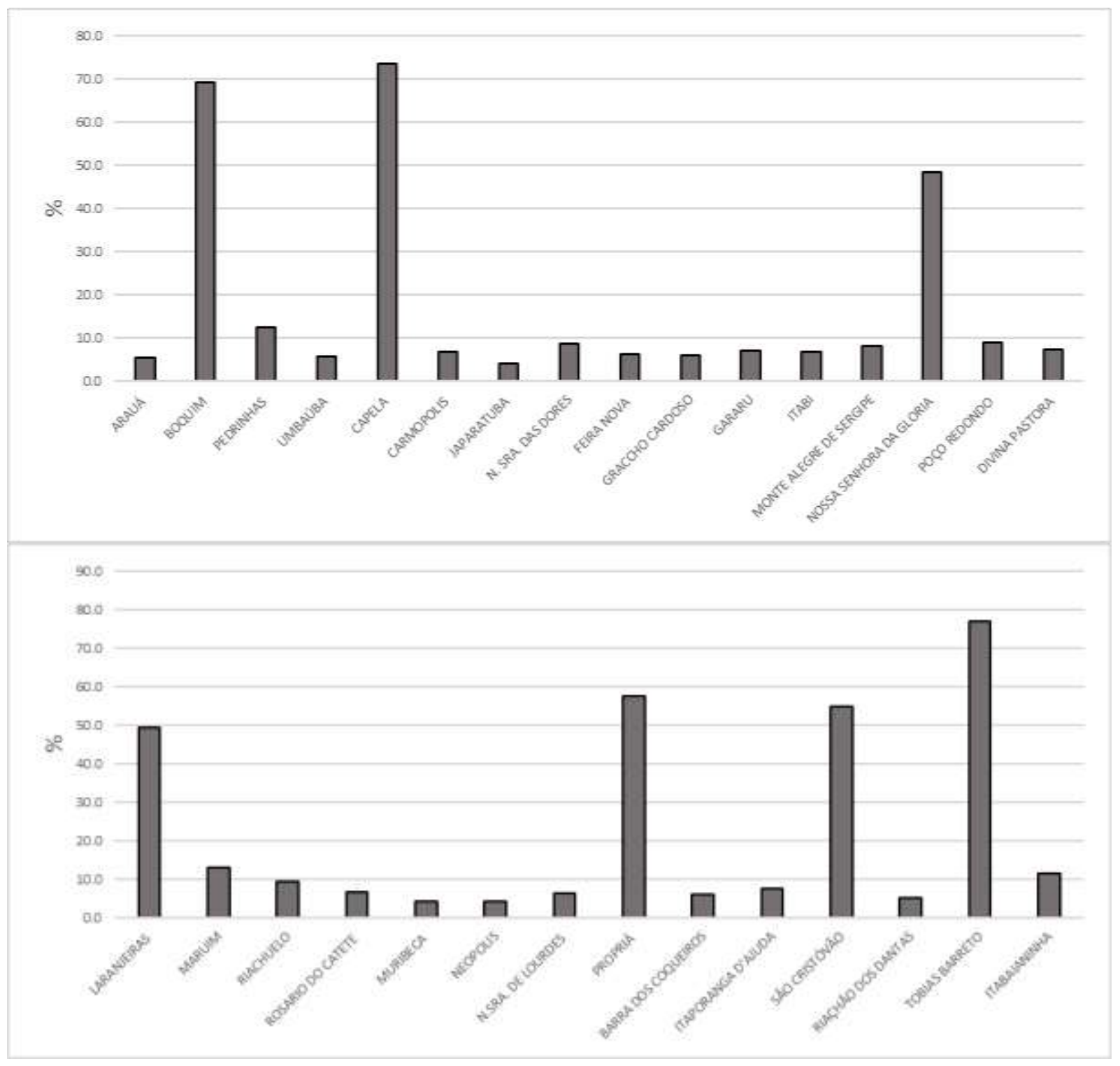

Fonte: FUNESA (2018).

\section{Discussão}

A Equidade é um dos princípios que regem o Sistema Único de Saúde - SUS. Esse princípio considera que as diferenças devem ser consideradas, ou seja, deve-se "tratar desigualmente os desiguais" para que as diferentes necessidades de cada grupo sejam atendidas. Diferente do que se entende por igualdade, a equidade pretende promover uma equiparação no que tange o acesso aos serviços de saúde, necessária quando se trata de pacientes com deficiência para que haja equiparação nas condições de acesso (Brasil, 2017; Castro et al., 2010).

Castro e colaboradores (2010) analisaram as dificuldades enfrentadas pela pessoa com deficiência ao acessar serviços de saúde. Foi observado que podem ser fatores negativos o tempo de espera no serviço - considerado um problema pela possível necessidade especial de alimentação, higiene e descanso por pacientes especiais, problemas com estacionamento, falta de rampas, elevadores, cadeiras de rodas e sanitários adaptados. Também foi observado que metade dos pacientes entrevistados 
necessitavam de um acompanhante para se deslocar até o serviço de saúde. Os autores concluíram que problemas que dificultam a acessibilidade da pessoa com deficiência aos serviços de saúde violam o princípio da Equidade, sendo a eliminação de tais problemas essenciais para proporcionar possibilidades igualitárias em relação as pessoas sem deficiências. Os fatores apontados tornam-se agravados pelo nível econômico da família ondem vivem as pessoas com deficiência em virtudes dos custos agregados para a realização dos tratamentos odontológicos em locais fora do município de residência.

Em Sergipe, 48.5370 das famílias cadastradas no CadÚnico 60\% possuem renda per capta de até $\mathrm{R} \$ 89,00$ mensais, 8\% possuem renda entre $\mathrm{R} \$ 89,01$ e 178,00, 17\% possuem renda per capta entre 178.01 e meio salário-mínimo e 15\% possuem renda per capta maior que meio salário-mínimo (Brasil C, 2020). Como demostrado na Tabela 1 nestas famílias encontram-se também pacientes com necessidades especiais. Observa-se que a maioria é SUS dependente e que diferentes coberturas de saúde bucal na atenção básica influenciam diretamente na atenção à saúde dessa população. Verifica-se também que há dificuldade do acesso aos serviços de saúde pela dificuldade para se deslocar para outro município em busca de uma atenção especializada.

Foi observado que mais de $70 \%$ dos encaminhamentos para atendimento nos CEOs estaduais de Sergipe foram de usuários residentes nas cidades-sede do serviço. Uma vez que foi encontrada correlação significativa entre a distância dos municípios até o CEO e população com renda per capita até $\mathrm{R} \$ 178,00$, a necessidade de deslocamento até o serviço pode ser um obstáculo para o acesso. Couto e colaboradores (2020) em estudo realizado no município de Aracaju SE observaram uma maior prevalência de atendimentos aos PNE residentes em bairros mais próximos aos locais onde estava localizado o Centro de Especialidades Odontológica. Resultados semelhante foi encontrado no município de Feira de Santana-BA (Santos, 2014), sendo ressaltado que pode ocorrer falta de informação dos profissionais ou grande fila de espera, além do transporte para os pacientes. Travassos e Martins (2004) abordam que um dos aspectos do acesso aos serviços de saúde é a acessibilidade geográfica, relacionada a distância, tempo de locomoção e custo da viagem.

No presente estudo foi observada uma correlação significativa entre municípios com maior número de pessoas com deficiência que recebem bolsa família e menor cobertura de saúde bucal na atenção básica. Desse modo, a baixa cobertura de saúde bucal na atenção básica pode ser um fator dificultador para o acesso do paciente especial ao tratamento nos Centros de Especialidades Odontológicas, visto que estes não devem funcionar como porta de entrada para o sistema de saúde. No estudo de Souto e colaboradores (2016), realizado região Extremo Sul da Bahia, foi verificado cobertura de saúde bucal na atenção básica variando de 57 a 132\%, no presente estudo, verifica-se que no Estado de Sergipe há municípios com menores coberturas em saúde bucal na atenção básica, como o município de Carira com 15\% de atenção. Assim o paciente por não ter acesso a atenção básica nunca será referenciado para a atenção especializada. Munícipios com baixa cobertura leva a uma desassistência da sua população.

Outro fator analisado no presente estudo foi a incidência de pobreza na população, no qual optou-se por utilizar o instrumento de vulnerabilidade social por meio da bolsa família e CadÚnico. A incidência de pobreza observada no estudo de Herkrath e colaboradores (2013) no Amazonas variou de 40 a 60\%. No estudo de Souto e colaboradores encontraram que especialistas são fatores que interferem nos resultados das metas e efetivamente na garantia do atendimento odontológico para as pessoas com deficiências, em particular numa população de baixa renda, economicamente desprovida de recursos.

\section{Conclusão}

Há dificuldade de acesso do paciente com necessidades especiais em termos de referenciamento e assistência em percentual de cobertura de saúde bucal.O baixo percentual de cobertura de saúde bucal e de referenciamento dificultam o 
acesso dos PNE à assistência odontológica. As condições socioeconômicas e demográficas interferem no acesso dos PNE aos CEOS em Sergipe.

\section{Agradecimentos}

Ao edital PPSUS - Sergipe (2018) ao edital Universal- CNPq (2018). A Secretaria Estadual de Sergipe e a FUNESA.

\section{Referências}

Brasil. Ministério da Saúde. Secretaria de Atenção À Saúde. Departamento de Atenção Básica. Coordenação Nacional de Saúde Bucal. Diretrizes da Política Nacional de Saúde Bucal, 2004. http://bvsms.saude.gov.br/bvs/publicacoes/politica_nacional_brasil_sorridente.htm

Brasil. Ministério da Saúde. Secretária de Atenção à Saúde. Departamento de Atenção Básica. Caderno de Atenção Básica. 2008. https://bvsms.saude.gov.br/bvs/publicacoes/saude_bucal.pdf

Brasil. Ministério da Saúde. Portaria n 599, de 23 de março de 2006. Define a implantação de Especialidades Odontológicas (CEOs) e de Laboratórios Regionais de Próteses Dentárias (LRPDs) e estabelecer critérios, normas e requisitos para seu credenciamento.

Brasil. Ministério da Saúde. Centro de Especialidades Odontológicas. Ministério da Saúde, 2017. https://www.saude.gov.br/acoes-e-programas/politicanacional-de-saude-bucal/atencao-especializada/centro-de-especialidades-odontologicas

Brasil A. Ministério da Saúde. Cadastro Nacional de Estabelecimento de Saúde. DATASUS. Ministério da Saúde. 2020. http://cnes.datasus.gov.br/pages/estabelecimentos/consulta.jsp

Brasil B. Ministério da Saúde. Secretária de Atenção Primária. Relatório Histórico de Cobertura. e-Gestor. Ministério da Saúde, 2020 https://egestorab.saude.gov.br/paginas/acessoPublico/relatorios/relHistoricoCobertura.xhtml

Brasil C. Ministério da Cidadania. Secretária de Avaliação e Informação. Cadastro Único. CECAD 2.0. Ministério da Saúde, 2020. https://aplicacoes.mds.gov.br/sagi/cecad20/painel03.php

Brasil. Ministério da Saúde. Secretaria de Atenção à Saúde. Implantação das Redes de Atenção à Saúde e Outras Estratégias da SAS. Brasília, DF: Ministério da Saúde, 2014. http://bvsms.saude.gov.br/bvs/publicacoes/implantacao_redes_atencao_saude_sas.pdf

Brasil. Ministério da Saúde. Decreto n ${ }^{\circ}$ 6135, de 26 de junho de 2007. Dispõe sobre o Cadastro Único para Programas Sociais do Governo Federal. Brasília, DF: Ministério da Saúde, 2014. http://www.planalto.gov.br/ccivil_03/_Ato2007-2010/2007/Decreto/D6135.htm

Brasil. Ministério da Saúde. Princípios do SUS. Saúde. Ministério da Saúde; 2017. https://www.saude.gov.br/sistema-unico-de-saude/principios-do-sus

Carreiro, D. L., Souza, G. S., Coutinho, L. M., Haikal, D. S., \& Martins, M. E. V. L. (2019). Acesso aos serviços odontológicos e fatores associados: estudo populacional domiciliar. Ciência \& Saúde Coletiva, 24(3):1021-1032.

Castro, S. S., Lefèvre, F., Lefèvre, A. M. C. \& Cesar, C. L. G. (2010). Acessibilidade aos serviços de saúde por pessoas com deficiência. Revista de Saúde Pública,45(1):99-105.

Costa, S. M., Nickel, D. A., Borges, C. M., Campos, A. C. V., \& Verdi M. I. M. (2012). Política Nacional de Saúde Bucal e bioética da proteção na assistência integral. Revista Bioética, 20(2):342-8.

Couto, G. R., Santo, M. A. L., Paiva, S. M., Prado, E. F. \& Amaral, R. C. (2021). Análise de desempenho da atenção odontológica especializada em rede de cuidados a pessoa com necessidades especiais. Research, Society and Development, 10 (2), e35710212678.

Herkrath, F. J., Herkrath, A. P. C. Q., Costa, L. N. B. S.\& Gonçalves, M. J. F. (2013). Desempenho dos Centros de Especialidades Odontológicas frente ao quadro sociodemográfico dos municípios do Amazonas, Brasil, 2009. Saúde debate,37( 96 ): 148-158.

IBGE - Instituto Brasileiro de Geografia e Estatística. Pesquisa Nacional por Amostra de Domicílios. IBGE. IBGE, 2013. https://www.ibge.gov.br/estatisticas/sociais/populacao/19897-sintese-de-indicadores-pnad2.html?edicao=18331\&t=sobre

Mendes, E. V. (2011). As redes de atenção à saúde. Organização Pan-Americana de Saúde. https://www.paho.org/bra/index.php?option=com_docman\&view=download\&category_slug=servicos-saude-095\&alias=1402-as-redes-atencao-a-saude-2aedicao-2\&Itemid=965.

Santos, C. (2014). Perfil epidemiológico dos pacientes com necessidades especiais atendidos em um centro de especialidades odontológicas do interior baiano. Revista Baiana de Saúde Pública,38(1):83-94.

Sergipe. Secretaria de Estado da Saúde. Fundação Estadual de Saúde. Centro de Especialidades Odontológicas. Protocolo dos Centros de Especialidades Odontológicas Estaduais. Editora Funesa; 2019. https://www.funesa.se.gov.br/wp-content/uploads/2019/02/Protocolo-de-Atendimento-CEOs.pdf

Souto, A. P. C., Bastos, B. C., Pimenta, R. M. C. \& Santos, L. P. S (2016). Avaliação da produtividade dos Centros de Especialidades Odontológicas do Extremo Sul da Bahia. Revista de Saúde Coletiva da UEFS,9,119-27.

Travassos, C., \&Martins, M.(2004). Uma revisão sobre os conceitos de acesso e utilização de serviços de saúde. Cad. Saúde Pública, $20(2)$, S190-S198. 